\title{
The ORFEUS II Echelle Spectrometer: Instrument description, performance and data reduction
}

\author{
J. Barnstedt ${ }^{1}$, N. Kappelmann ${ }^{1}$, I. Appenzeller ${ }^{2}$, A. Fromm ${ }^{1}$, M. Gölz ${ }^{1}$, M. Grewing ${ }^{1,3}$, W. Gringel ${ }^{1}$, C. Haas ${ }^{1}$, \\ W. Hopfensitz ${ }^{1}$, G. Krämer ${ }^{1}$, J. Krautter ${ }^{2}$, A. Lindenberger ${ }^{1}$, H. Mandel ${ }^{2}$, and H. Widmann ${ }^{1}$ \\ 1 Institut für Astronomie und Astrophysik, Abt. Astronomie, Eberhard-Karls-Universität Tübingen, Waldhäuserstr. 64, \\ D-72076 Tübingen, Germany \\ 2 Landessternwarte Heidelberg, Königstuhl, D-69117 Heidelberg, Germany \\ 3 Institut de Radio Astronomie Millimétrique (IRAM), 300 rue de la Piscine, 38406 Saint Martin d'Hères, France
}

Received May 20; accepted September 15, 1998

\begin{abstract}
During the second flight of the ORFEUS-SPAS mission in November/December 1996, the Echelle spectrometer was used extensively by the Principal and Guest Investigator teams as one of the two focal plane instruments of the ORFEUS telescope. We present the inflight performance and the principles of the data reduction for this instrument. The wavelength range is $90 \mathrm{~nm}$ to $140 \mathrm{~nm}$, the spectral resolution is significantly better than $\lambda / \Delta \lambda=10000$, where $\Delta \lambda$ is measured as FWHM of the instrumental profile. The effective area peaks at $1.3 \mathrm{~cm}^{2}$ near $110 \mathrm{~nm}$. The background is dominated by straylight from the Echelle grating and is about $15 \%$ in an extracted spectrum for spectra with a rather flat continuum. The internal accuracy of the wavelength calibration is better than $\pm 0.005 \mathrm{~nm}$.
\end{abstract}

Key words: instrumentation: spectrographs - space vehicles — stars: individual: HD 93521 - ultraviolet: general

\section{Introduction}

The German spacecraft ORFEUS-SPAS was the primary payload aboard the Space Shuttle Columbia during mission STS-80, flown from 19 November to 7 December, 1996. The ORFEUS telescope was the main instrument aboard the astronomy platform Shuttle Pallet Satellite (ASTRO-SPAS).

The ASTRO-SPAS is a free flying platform that is designed to operate autonomously in the vicinity (up to $60 \mathrm{~km}$ ) of the Space Shuttle for a limited period of up to

Send offprint requests to: J. Barnstedt e-mail: barnstedt@astro.uni-tuebingen.de
14 days. It was designed by the Daimler-Benz Aerospace AG (DASA) in Ottobrunn, Germany.

With a mission duration of 17.7 days STS-80 was the longest shuttle flight to date, the free flying time of ORFEUS-SPAS was 14 days. ORFEUS-SPAS was released a few hours after launch on 20 November, 1996. It was the third flight of the reusable ASTRO-SPAS platform and the second flight of the ORFEUS-SPAS configuration.

The ORFEUS telescope (Orbiting and Retrievable Far and Extreme Ultraviolet Spectrometer) (Krämer et al. 1988, 1990; Grewing et al. 1991, 1992) consists of a $1 \mathrm{~m}$ normal incidence mirror with a focal length of $2.4 \mathrm{~m}$ and two spectrometers as focal plane instrumentation: the Berkeley spectrograph and the Echelle spectrograph. The Berkeley spectrograph was developed by the Space Sciences Laboratory of the University of California at Berkeley (Hurwitz \& Bowyer 1986, 1996; Hurwitz et al. 1998) and the Echelle spectrograph was developed by the University of Tübingen and the Landessternwarte Heidelberg (Appenzeller et al. 1988). As a third independent instrument, the Interstellar Matter Absorption Profile Spectrometer (IMAPS) was attached to the ASTRO-SPAS (Jenkins et al. 1996).

The ORFEUS telescope and the Echelle spectrometer were designed and built by Kayser-Threde $\mathrm{GmbH}$ in Munich, Germany. The Echelle detector as well as the Echelle electronics and the Echelle onboard processor were designed and built by the Institute for Astronomy and Astrophysics, Department Astronomy, of the University of Tübingen.

The ORFEUS-SPAS total instrument operation time was $263^{\mathrm{h}} .0$, while the net integration time was $164^{\mathrm{h}} .9$, resulting in an efficiency of $62.5 \%$ for all instruments. This is an extremely high value as compared to other satellite missions. 


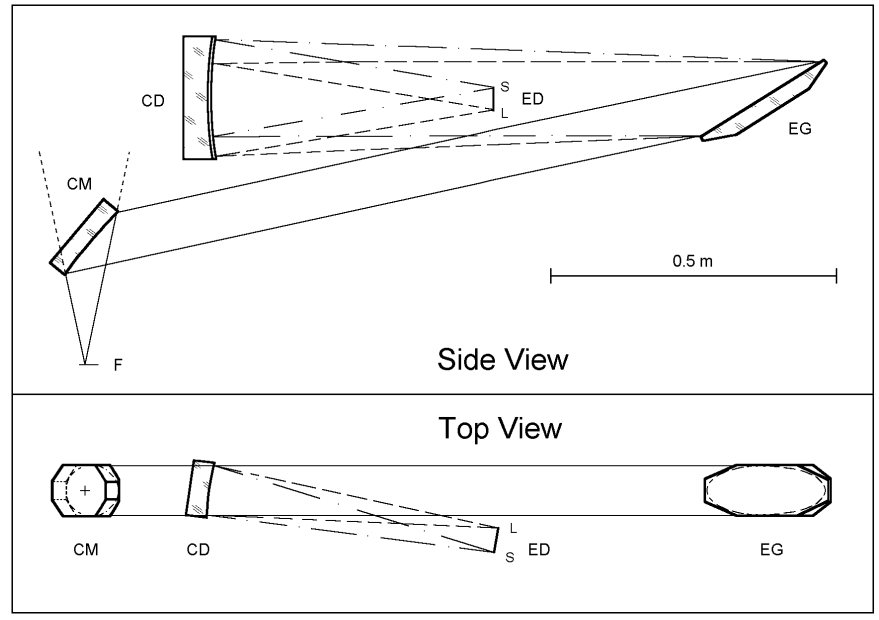

Fig. 1. Optical design of the Echelle spectrometer. Legend: $\mathrm{F}=$ telescope focus and entrance diaphragm, $\mathrm{CM}=$ collimator mirror, $\mathrm{EG}=$ Echelle grating, $\mathrm{CD}=$ cross disperser, $\mathrm{ED}=$ Echelle detector, $\mathrm{L}=$ long wavelength side, $\mathrm{S}=$ short wavelength side

$50 \%$ of the total integration time were reserved for Guest Investigators selected by peer review. The other $50 \%$ were used by the Principal Investigator teams who had provided the instruments. 65 distinct objects were observed with the Echelle spectrometer.

In this paper we present the performance figures for the Echelle spectrometer and a short description of the principles of the data extraction procedure.

\section{Instrument description}

Within the ORFEUS telescope a movable mirror is used to switch between the two spectrometers. We will restrict the instrument description to the Echelle spectrometer.

The movable mirror is designed to act at the same time as an off-axis parabolic collimator (CM, Fig. 1). When moved in it feeds the Echelle grating (EG) with a parallel light bundle. When the collimator mirror is moved out, the beam is falling directly into the Berkeley spectrometer.

The Echelle grating has a groove density of 316 lines $/ \mathrm{mm}$ with a blaze angle of 62.5 . It is operated in the diffraction orders 40 to 61 covering the wavelength range from $90 \mathrm{~nm}$ to $140 \mathrm{~nm}$.

The Echelle diffraction orders are separated by means of a spherical cross disperser grating (CD) with 1200 lines/mm which also serves as the spectrograph camera. This design minimizes the number of reflections, which, in view of the low reflectivities in the FUV (ranging from $10 \%$ to $30 \%$ ), was of critical importance for achieving an acceptable overall efficiency.

The spectrum is recorded by the Echelle detector (ED), which has a plane surface and a sensitive area of $40 \mathrm{~mm} \times 40 \mathrm{~mm}$.
The Echelle spectrometer is designed to achieve a spectral resolution of $\lambda / \Delta \lambda=10000$ when used with an entrance aperture of $10^{\prime \prime}$ diameter (Appenzeller et al. 1988).

\subsection{The Echelle detector}

The Echelle detector is a photon counting microchannel plate detector with a wedge and strip readout system. Three stacked microchannel plates (MCPs), operated in a Z-configuration, provide a gain of $10^{7}$ to $10^{8}$ electrons/photon. Each of the three MCPs has its own power supply and an accelerating voltage is applied to the gaps between the MCPs. During flight the gain of the Echelle detector was monitored and could be controlled by variation of the high voltage of the third MCP. The high voltage could be adjusted by commands during flight or by starting an automatic function that adjusted the high voltage so that a predefined gain value was achieved.

A repeller grid in front of the MCPs produces an electric field of about $50 \mathrm{~V} / \mathrm{mm}$, which is used to force those photo electrons back into the MCP channels, which are released from the areas in between the channels. This improves the quantum efficiency by about $30 \%$ but also causes a loss of $10 \%$ due to the shading by the repeller grid.

A four electrode wedge-and-strip anode (Martin et al. 1981) was used as readout system for the MCP detector. A special design allowed for a significant reduction of the edge distortions, which are basically unavoidable. Further, by oversizing the active area of the anode to $44 \mathrm{~mm} \times 44 \mathrm{~mm}$, it was possible to avoid any distortions within the sensitive area of the detector. The image format of the detector is 1024 pixels by 512 pixels, which corresponds to the active area of the anode.

The detector sends for each photon event $x / y$-coordinates to the Echelle onboard processor where these events are integrated onto an image memory. The content of this image memory is stored on magnetic tape at the end of each observation.

In parallel, the $x / y$-coordinates for each single photon event are stored on tape directly, thus keeping also the photon arrival time to an accuracy of better than $1 \mathrm{~s}$. This method is limited by the ASTRO-SPAS interface to an event rate of less than 3500 counts/s, whereas the onboard integration works with much higher photon rates up to 30000 counts/s. The electronic dead time is about $13 \mu \mathrm{s}$ per event.

The count rate of the Echelle detector is monitored online and also stored on tape together with many other housekeeping data. This count rate is derived from all events registered by the charge amplifiers falling above the lower threshold of the detector electronics. Not all of these events are finally integrated into the image for the following reasons: 
- An upper electronic threshold suppresses pulses too large to be processed by the position decoding electronics.

- At high count rates the dead time of $13 \mu \mathrm{s}$ for position decoding reduces the rate of registered events accordingly.

- Dead time effects from the interface electronics and from the onboard processor further reduce the electronic efficiency.

These effects are the cause that in general the rate of the integrated counts within an image is between $15 \%$ and $25 \%$ lower than the count rate of the lower electronic threshold.

In the main dispersion direction somewhat more than 3 pixels correspond to one optical resolution element of $\Delta \lambda=\lambda / 10000(161 \mu \mathrm{m})$. The electronic resolution was estimated to be about 1.5 detector pixels FWHM. So the electronic detector resolution is sufficient to maintain the optical resolution of the Echelle spectrometer.

Electronic test pulses were fed onto the anode with a frequency of $10 \mathrm{~Hz}$ at two different detector positions and with 3 different pulse heights. These test pulses were extremely useful during the checkout of the instrument and provide a means of monitoring the electronic performance of the detector throughout the mission.

\section{Extraction of Echelle orders}

The extraction of the Echelle spectra from the Echelle detector images in $x$-direction (main dispersion direction) is done by summing up 11 pixels (orders 40 to 49) or 9 pixels (orders 50 to 61 ) in $y$-direction. The center of the extraction in $y$-direction follows a straight line along each order, but is located on integer pixel numbers.

Some Echelle images show tilted absorption lines within the strip of the Echelle orders. The reason for this effect is still unclear. In such cases the extraction was done by summing up the pixels tilted by 45 degrees, which results in a significant increase in resolution. However an additional wavelength error was introduced by this procedure since the observed wavelength in this way became dependent on the centering of the Echelle order within the extraction strip. This is a nonlinearity error of the size of one pixel, which corresponds to $1 / 3$ of the optical resolution element and is equal to $\Delta \lambda=\lambda / 30000$.

\subsection{Background}

Between the Echelle orders a 3 pixel wide area was used to estimate the background. With exception of orders 40,41 and 42 the background was calculated as average of the strip above and below the corresponding order. For the first three orders only the background values below each order were calculated. The so calculated background was smoothed prior to being used for subtraction.

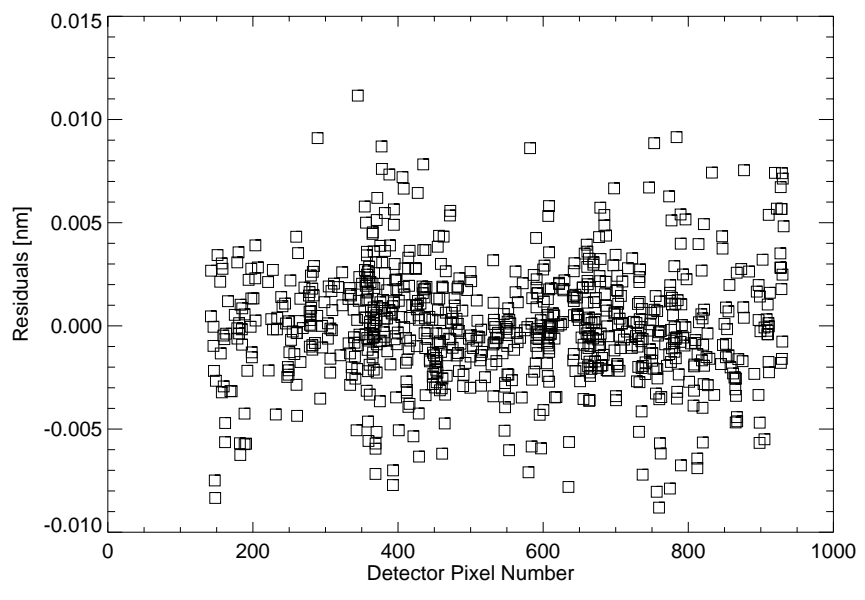

Fig. 2. Residuals of the wavelength calibration fit. 814 positions of interstellar absorption lines from 12 different objects were used to fit 7 wavelength calibration parameters

The intrinsic background of the detector was about $10^{-5}$ counts/s/pix. As 9 or 11 detector pixels are added to one spectral pixel, this corresponds to about $10^{-4}$ counts/s/pix in the extracted spectra, which is negligible.

By far the strongest contribution to the background is straylight from the Echelle grating. The contribution in counts/detector-pixel is about $5 \%$ to $10 \%$ for the low Echelle orders and $10 \%$ to $20 \%$ for the high Echelle orders as these are closer together. The percentage values refer to the maximum counts/detector-pixel values of the Echelle orders. The contribution in counts/spectrum-pixel is about $15 \%$ for spectra with a rather flat continuum.

The Echelle straylight is scattered exactly horizontally across the detector, whereas the spectral orders run slightly tilted across the detector corresponding to the dispersion of the cross disperser. Hence emission lines produce additional straylight while broad absorption lines lead to a reduced straylight contribution in a horizontal line at the $y$-position of this absorption line. These varying straylight contributions are not yet correctly handled by the background subtraction algorithm.

An additional contribution is caused by particle events related to the South Atlantic Anomaly (SAA). These produced count rates of up to 1000 counts/s resulting in up to $210^{-3}$ counts/s/pix on the detector and $210^{-2}$ counts/s/pix in the spectra. The maximum duration of the passage through the SAA was about $12 \mathrm{~min}$ and the count rate curve showed a quasi Gaussian shape during this time. In the case of weak targets where the SAA background reduces the signal to noise ratio of the spectrum significantly, we are able to integrate the spectrum from the single photon records excluding the SAA transit period. 


\subsection{Correction of the blaze function}

The efficiency of blazed Echelle gratings varies as $(\sin (x) / x)^{2}$ (blaze function) (Schroeder \& Hiliard 1980), where $\mathrm{x}$ depends on the deviation of the output angle from the specular angle of maximum efficiency (blaze angle) and on the wavelength. The optimized diffraction direction was preflight adjusted to the $y$-centerline of the Echelle detector. In the flight data we found the center of maximum efficiency to differ between the Echelle orders and furthermore both the position and the width of the blaze function differing from observation to observation. Therefore it was necessary to introduce an individual blaze correction for each observation. For spectra that showed a relatively undisturbed continuum the blaze correction could be done with a rather high accuracy. However for many spectra which are dominated by absorption lines only the overlap region between two adjacent orders could be used as a criterion for a good blaze correction, resulting in a less reliable correction.

After completion of the blaze correction for all observations no systematic time dependent effects in the variation of the blaze function were found.

\subsection{Wavelength calibration}

The wavelength calibration was calculated from the positions of 814 interstellar absorption lines in the Echelle images of 12 different objects. The centers of the lines were determined by fitting Gaussian functions to the profiles using a preliminary wavelength calibration. After identification of the lines a table with laboratory wavelengths and corresponding detector pixel numbers was created. Using the preliminary wavelength calibration, a mean radial velocity component for each object was estimated and corrected for. This approach assumes, that for each individual object all identified interstellar absorption lines, mainly $\mathrm{H}_{2}$ and neutral elements, originate in the same volume of gas.

With a least square fit algorithm we then determined a new set of pixel to wavelength conversion parameters.

In Fig. 2 the residuals for all lines demonstrate that the mean accuracy of the wavelength correspondence is better than $\pm 0.005 \mathrm{~nm}$, i.e. better than the optical resolution of the instrument.

The above procedure results in a relative wavelength calibration. In order to determine an absolute scale we used the position of the geocoronal Ly- $\alpha$ emission line as a reference. We found that for different observing blocks the position of the Ly- $\alpha$ line changed by up to $0.006 \mathrm{~nm}$ (Fig. 3). The reason for this shift is still unclear, we assume that it emerges from temperature drifts of the telescope and the Echelle spectrometer. A correction for this effect has been applied in each observing block.

Shifting of the Echelle spectrum on the detector surface (e.g. displacement of the target within the aperture)

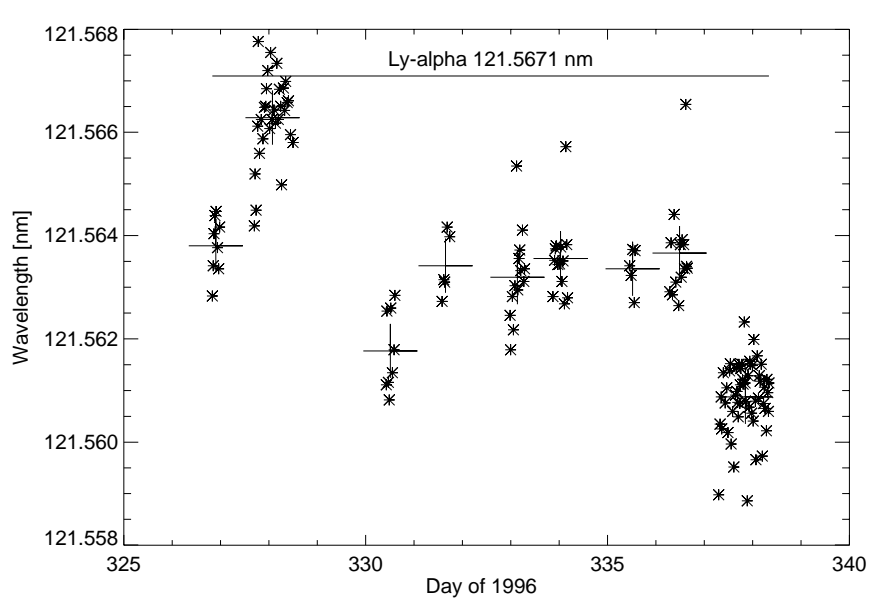

Fig. 3. Apparent wavelengths of the geocoronal Ly- $\alpha$ emission line plotted versus time of observation. For each of the 9 observing blocks for the Echelle spectrometer the large crosses mark the average Ly- $\alpha$ position, which was later used for correction of the time dependent wavelength shift. For these measurements no wavelength corrections except for the satellite's orbital velocity were applied

basically results in a relative wavelength shift and thus in a radial velocity shift rather than in a wavelength shift. The reason is, that the wavelength dispersion is proportional to the wavelength.

The wavelength scale of the targets were shifted to correct for radial velocity components due to the Earth's movement (heliocentric correction) and due to the satellite's orbital movement.

A further shift occurs when the target image is not exactly centered within the $20^{\prime \prime}$ diaphragm. The maximum resulting uncertainty due to the $10^{\prime \prime}$ radius of the diaphragm is $\pm 1.210^{-4}$ as a relative wavelength error (corresponding to $\pm 36 \mathrm{~km} \mathrm{~s}^{-1}$ ). This error was not corrected up to now.

\subsection{Correction of loss of sensitivity in the detector edges}

In the corners of the detector area and at the left edge the efficiency of the repeller grid is reduced, probably due to an inhomogenous field close to the edge of the detector. A step occurs between lower and normal sensitivity. In some images a circularly shaped area is visible due to this effect. We estimated a loss of about $25 \%$ and corrected this by applying a "smooth" step function. The position, width and height of the step was estimated for each order from the sum of all Echelle measurements.

A detailed flat field correction was not applied, due to the fact, that the optical light path of the spectrometer cannot be reproduced in our laboratory. This however would be essential for an exact estimation of the flat field behavior of the detector. Any other correction methods are too uncertain to be useful. 


\subsection{Absolute flux calibration}

We used an HST archive model of G191B2B "http:// www.stsci.edu/ftp/cdbs/calspec/g191b2b_mod_002.tab" as a reference for the absolute flux calibration. The calibration was crosschecked with a model of BD $+28^{\circ} 4211$ (R. Napiwotzki 1997, priv. comm.). We estimate an accuracy of $\pm 10 \%$ for the flux calibration, provided the object was fully centered within the aperture, which was not always the case.

\section{Performance}

\subsection{Sensitivity}

The total efficiency of the Echelle spectrometer was estimated before the flight by measuring the efficiencies of the individual optical components. The product of these values resulted in a maximum effective area of $2.4 \mathrm{~cm}^{2}$.

For postflight recalibration we used a HST calibration spectrum of G191 B2B. By comparing the fluxes within complete orders we estimated an effective area value for the central wavelength of each Echelle order. Values for Echelle orders that were contaminated by strong absorption lines (e.g. Ly- $\alpha$ ) were deleted from the data set. This procedure leads to a maximum effective area of $1.3 \mathrm{~cm}^{2}$ near $110 \mathrm{~nm}$ (Fig. 4), which is based on all detector counts above the lower electronic threshold.

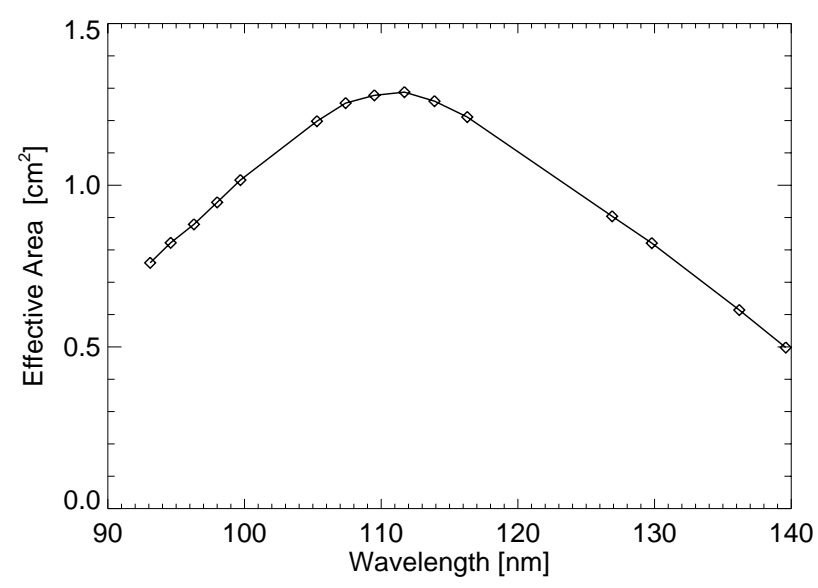

Fig. 4. Effective area curve for the Echelle spectrometer. The symbols denote the center wavelengths of those Echelle orders for which a fit value to the HST model spectrum was estimated

The $20^{\prime \prime}$ diameter of the diaphragm should be sufficient to maintain the total flux of the object, as the jitter of the ASTRO-SPAS was about $\pm 2^{\prime \prime}$. But we have indications that the telescope was not precisely focused during some periods of the mission, due to thermal problems during unexpected flight modes of the ASTRO-SPAS. Together with the fact that some objects were not fully centered this led to significant nonstatistical variations of the count rates, which resulted in a reduction of the flux and thus sensitivity. Therefore the flux calibration was calculated for the maximum observed count rate for the corresponding target (also from other observations of this target, if necessary). This count rate was scaled with the registered count rate in the integrated image.

\subsection{Spectral resolution}

The spectrum of HD 93521 with a signal to noise ratio of 20 to 30 is best suited to study the spectral resolution of the Echelle spectrograph. It shows many interstellar absorption lines, mainly from molecular hydrogen, which are very sharp and rather strong. Among a total of 814 interstellar line positions used for wavelength calibration, 159 line positions originated from this object. We coadded two observations of this object with integration times of $1080 \mathrm{~s}$ and $660 \mathrm{~s}$ respectively, taken within two successive orbits (Fig. 5).

We fitted many of these absorption lines in the wavelength range from $96 \mathrm{~nm}$ to $110 \mathrm{~nm}$ with Gaussian profiles and calculated the ratio of wavelength to the FWHM of these lines. We got values mainly between $\lambda / \Delta \lambda=7000$ to 10000 with depths of $60 \%$ to $80 \%$ (Fig. 6 and Table 1 ).

We further estimated an upper limit for the width of the instrument profile: Assuming that the absorption lines had originally depths of $100 \%$ with the same equivalent width, we calculated the width of the instrument profile that would be necessary to reproduce the observed absorption line profiles. For simplicity we assumed Gaussian profiles for both the absorption lines and the instrumental profile and a quadratic addition of the widths. The resulting values for the FWHM of the instrumental profiles are in the range $\lambda / \Delta \lambda=9000$ to 13000 (Table 1 ). As these calculations are estimates of a lower limit of the resolution of the Echelle spectrometer, we conclude that the achieved resolution of the Echelle spectrometer was significantly better than the goal of $\lambda / \Delta \lambda=10000$.

Another approach to estimate the achieved instrumental resolution is shown in Fig. 7. The equivalent widths of the fitted absorption lines are plotted against their observed $\lambda / \Delta \lambda$ values. A linear least square fit is applied to all data points (point No. 9 was excluded from the fit in a conservative approach). Extrapolating this fitted straight line to an equivalent width of 0 should reproduce the instrumental resolution. The result is a value between $\lambda / \Delta \lambda=11000$ and 12000 .

This result is not really surprising, as the spectral resolution of 10000 was calculated for a fully illuminated $10^{\prime \prime}$ diaphragm. Though the Echelle spectrometer was operated with a $20^{\prime \prime}$ diaphragm the ASTRO-SPAS pointing jitter was $\pm 2^{\prime \prime}$ and therefore the effective object image size was much smaller than the assumed $10^{\prime \prime}$. 


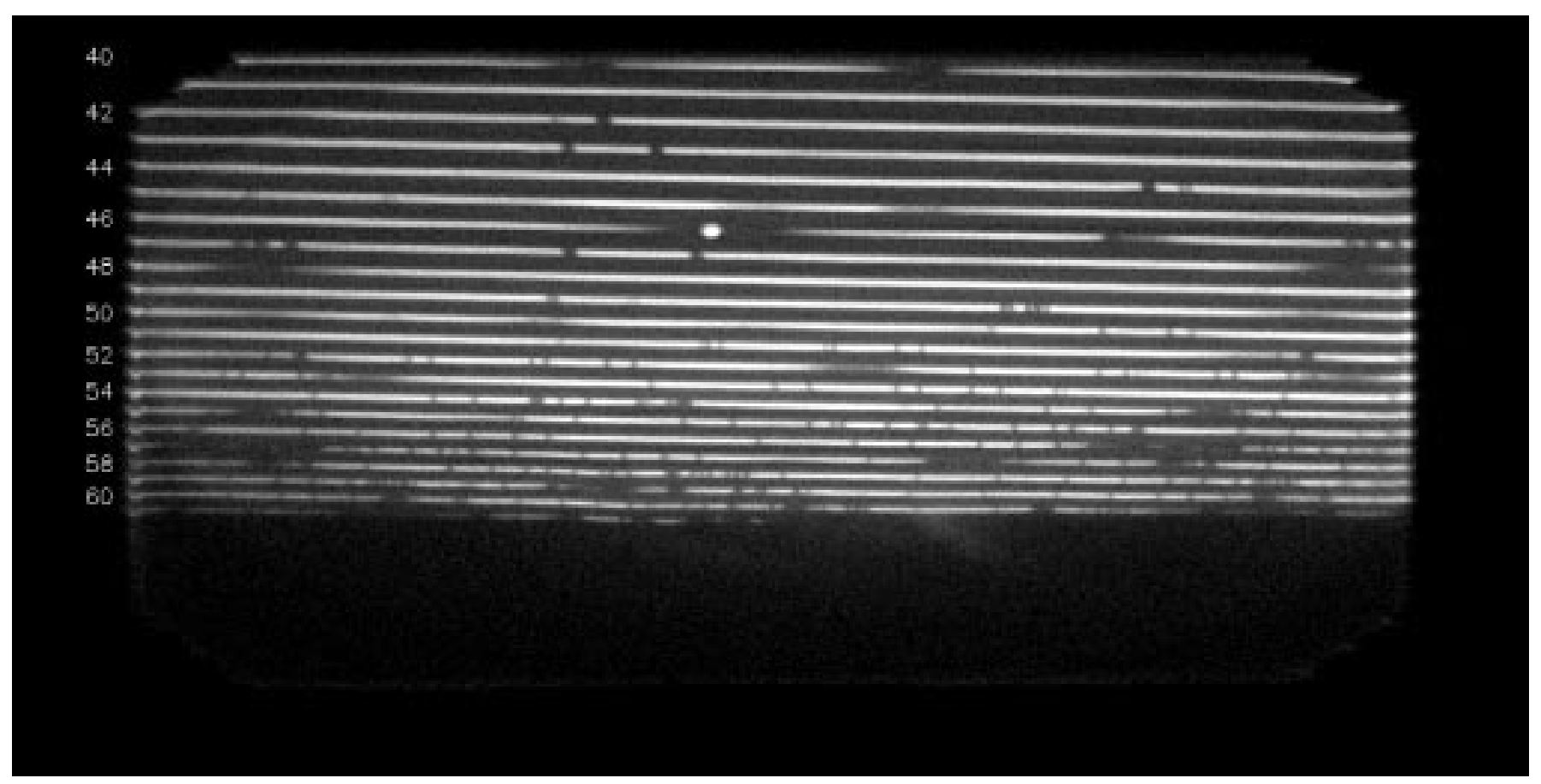

Fig. 5. Echelle image of HD 93521. The numbers at the left side denote the Echelle orders. The image is shown in the electronic format with $1024 \times 512$ pixels, which does not correspond to the square format of the detector of $40 \mathrm{~mm} \times 40 \mathrm{~mm}$. The Echelle spectrometer could show some more Echelle orders than the visible orders 40 to 61 , but below the Lyman limit at $91.15 \mathrm{~nm}$ all light is generally absorbed by interstellar hydrogen, so that none of the spectra contains any useful flux below this limit. (Orientation: long wavelengths are at top and at left side)

Table 1. Fitted Gaussian absorption profiles from the spectrum shown in Fig. 6. From the estimated equivalent widths a Gaussian instrument profile was calculated under the assumption that the original absorption line had an depth of $100 \%$. The broad stellar features are fitted for a better reproduction of the continuum, as the fitting algorithm used supports only a linear continuum

\begin{tabular}{|c|c|c|c|c|c|c|c|c|c|}
\hline No. & {$[\mathrm{nm}]$} & Identification & Shift & Depth & $\begin{array}{l}\Delta \lambda \\
(\mathrm{FWHM}) \\
{[\mathrm{nm}]}\end{array}$ & $\lambda / \Delta \lambda$ & $\begin{array}{l}\text { Equival. } \\
\text { Width } \\
{[\mathrm{nm}]}\end{array}$ & $\begin{array}{l}\Delta \lambda \text { instr. } \\
(\mathrm{FWHM}) \\
{[\mathrm{nm}]}\end{array}$ & $\begin{array}{l}\lambda / \Delta \lambda \\
\text { (instr.) }\end{array}$ \\
\hline 1 & 100.830 & $\mathrm{H}_{2} 100.8392$ & -0.009 & 82.4 & 0.01653 & 6099 & 0.01449 & 0.00937 & 10755 \\
\hline 2 & 100.841 & $\mathrm{H}_{2} 100.8553$ & -0.014 & 82.1 & 0.01538 & 6556 & 0.01343 & 0.00879 & 11470 \\
\hline 3 & 100.891 & $\mathrm{H}_{2} 100.903$ & -0.012 & 71.1 & 0.01150 & 8775 & 0.00869 & 0.00809 & 12470 \\
\hline 4 & 100.948 & Fe II 100.9580 & -0.010 & 21.4 & 0.00926 & 10907 & 0.00210 & 0.00904 & 11164 \\
\hline 5 & 100.966 & $\mathrm{H}_{2} 100.9772$ & -0.011 & 76.2 & 0.01561 & 6469 & 0.01265 & 0.01012 & 9981 \\
\hline 6 & 100.960 & stellar feature & & & & & & & \\
\hline 7 & 101.003 & $\mathrm{H}_{2} 101.0132$ & -0.010 & 68.3 & 0.01155 & 8756 & 0.00840 & 0.00843 & 11978 \\
\hline 8 & 101.083 & $\mathrm{H}_{2} 101.0941$ & -0.011 & 71.2 & 0.01243 & 8133 & 0.00942 & 0.00873 & 11583 \\
\hline 9 & 101.207 & $\mathrm{H}_{2} 101.2173$ & -0.010 & 48.0 & 0.00854 & 11845 & 0.00436 & 0.00750 & 13499 \\
\hline 10 & 101.214 & stellar feature & & & & & & & \\
\hline 11 & 101.259 & $\mathrm{H}_{2} 101.2681$ & -0.009 & 76.3 & 0.01395 & 7256 & 0.01132 & 0.00903 & 11217 \\
\hline 12 & 101.271 & $\mathrm{H}_{2} 101.2822$ & -0.011 & 67.3 & 0.01176 & 8611 & 0.00843 & 0.00869 & 11648 \\
\hline 13 & 101.314 & Fe II 101.3262 & -0.012 & 20.9 & 0.00966 & 10490 & 0.00214 & 0.00945 & 10726 \\
\hline 14 & 101.332 & $\mathrm{H}_{2} 101.3434$ & -0.011 & 69.5 & 0.01507 & 6726 & 0.01114 & 0.01084 & 9350 \\
\hline 15 & 101.422 & $\mathrm{H}_{2} 101.4334$ & -0.011 & 70.7 & 0.01521 & 6669 & 0.01144 & 0.01075 & 9431 \\
\hline 16 & 101.440 & $\mathrm{H}_{2} 101.4509$ & -0.011 & 63.4 & 0.01306 & 7765 & 0.00881 & 0.01010 & 10040 \\
\hline
\end{tabular}




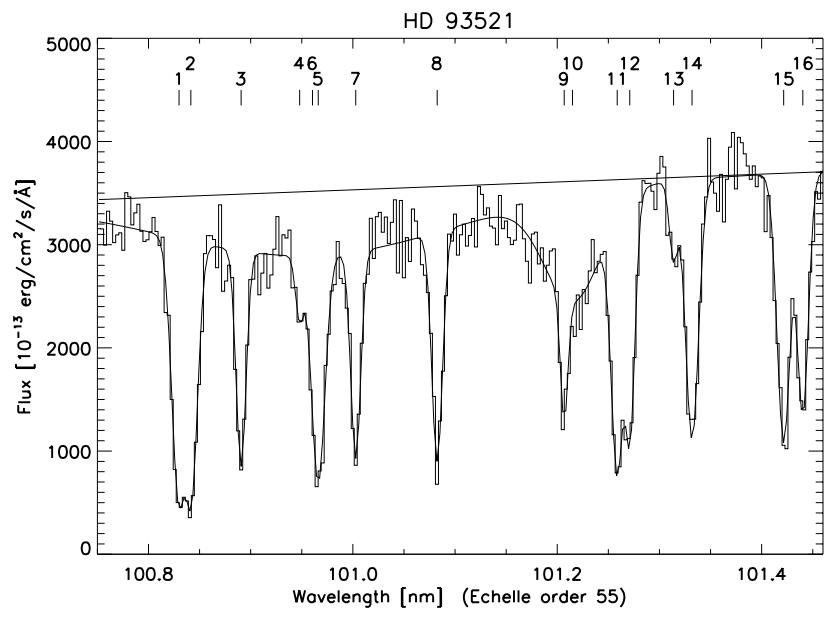

Fig. 6. Part of the Echelle spectrum of HD 93521. This demonstrates the resolution achieved with the Echelle spectrometer. The spectrum is plotted without any smoothing. The smooth curve plotted over the spectrum is a fit with several Gaussian shaped absorption profiles. The straight line shows the continuum used by the fitting algorithm. The continuum is corrected by additional broad absorption profiles (6 and 10). See Table 1 for the parameters of the fitted curves

Figure 8 shows the complete Echelle spectrum of HD 93521 smoothed with a 11 pixel wide boxcar. The individual Echelle orders are plotted separately, the spectral range for each order is indicated at the top of the figure. The overlap region between adjacent orders increases with the order number. A small gap exists between orders 40 and 41 and between orders 41 and 42 .

\section{Summary}

The Echelle spectrometer was one of the two focal instruments of the ORFEUS telescope, which was flown on its second mission in November/December 1996. The in-flight performance and the principles of data reduction for this instrument are presented. The wavelength range is $90 \mathrm{~nm}$ to $140 \mathrm{~nm}$ and we showed, that the spectral resolution is significantly better than $\lambda / \Delta \lambda=10000$, where $\Delta \lambda$ is measured as the FWHM of the instrumental profile. The effective area peaks at $1.3 \mathrm{~cm}^{2}$ near $110 \mathrm{~nm}$. The background is dominated by straylight from the Echelle grating and is about $15 \%$ in an extracted spectrum for spectra with a rather flat continuum. The internal accuracy of the wavelength calibration is better than $\pm 0.005 \mathrm{~nm}$. No corrections could be applied which would correct for a possible systematic shift of the object within the $20^{\prime \prime}$ aperture of the telescope.

For the future we plan to improved the data quality by further reduction steps and investigations:

- Integration of single photons with correction of actual orbital radial velocity and possibly correction for the ASTRO-SPAS pointing jitter.

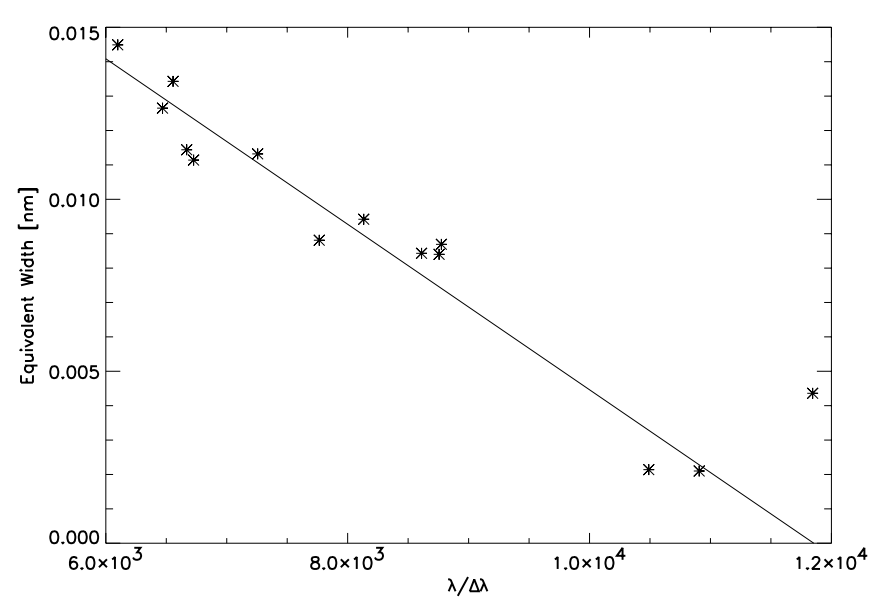

Fig. 7. Equivalent widths of fitted lines plotted against their observed $\lambda / \Delta \lambda$ values (Table 1 ). The straight line is a least square fit to the data points, except for absorption line No. 9 , which was excluded in a conservative approach. Extrapolating the linear fit to an equivalent width of 0 the observed line width should reproduce the instrumental width. The values shown indicate an instrumental resolution between 11000 and 12000

- Correlation of count rate variations with ASTROSPAS pointing jitter to estimate the object's position within the aperture and the size and the shape of the telescope's image of the object.

- Investigation of small scale flat field variations.

- Investigation of the cause for the "tilted" absorption lines within the spectral orders.

- Stray light model for improved background subtraction.

Acknowledgements. This project could not have been successfully carried out without the very efficient cooperation of all the many individuals and institutions involved. In particular we acknowledge the excellent performance of the teams of DASA with the construction and mission operations of the ASTROSPAS satellite, of Kayser-Threde with the construction of the ORFEUS telescope, the ground support teams at the Kennedy Space Center, the crew of STS-80 Columbia, the mission representatives of DARA and NASA and last not least our colleagues at the Space Sciences Laboratory of the University of California at Berkeley. We wish to thank Dr. Ralf Napiwotzki for calculating the atmosphere models for $\mathrm{BD}+28^{\circ} 4211$ and making them available to us. The ORFEUS program was supported by DARA grants WE3 OS 8501 and WE2 QV 9304 and NASA grant NAG5-696.

\section{References}

Appenzeller I., Krautter J., Mandel H., et al., 1988, in: A Decade of UV Astronomy with IUE, ESA SP-281, 2, 337

Grewing M., Krämer G., Appenzeller I., et al., 1991, in: Extreme Ultraviolet Astronomy, Malina R.F., Bowyer S. (eds.). Pergamon Press, p. 422 


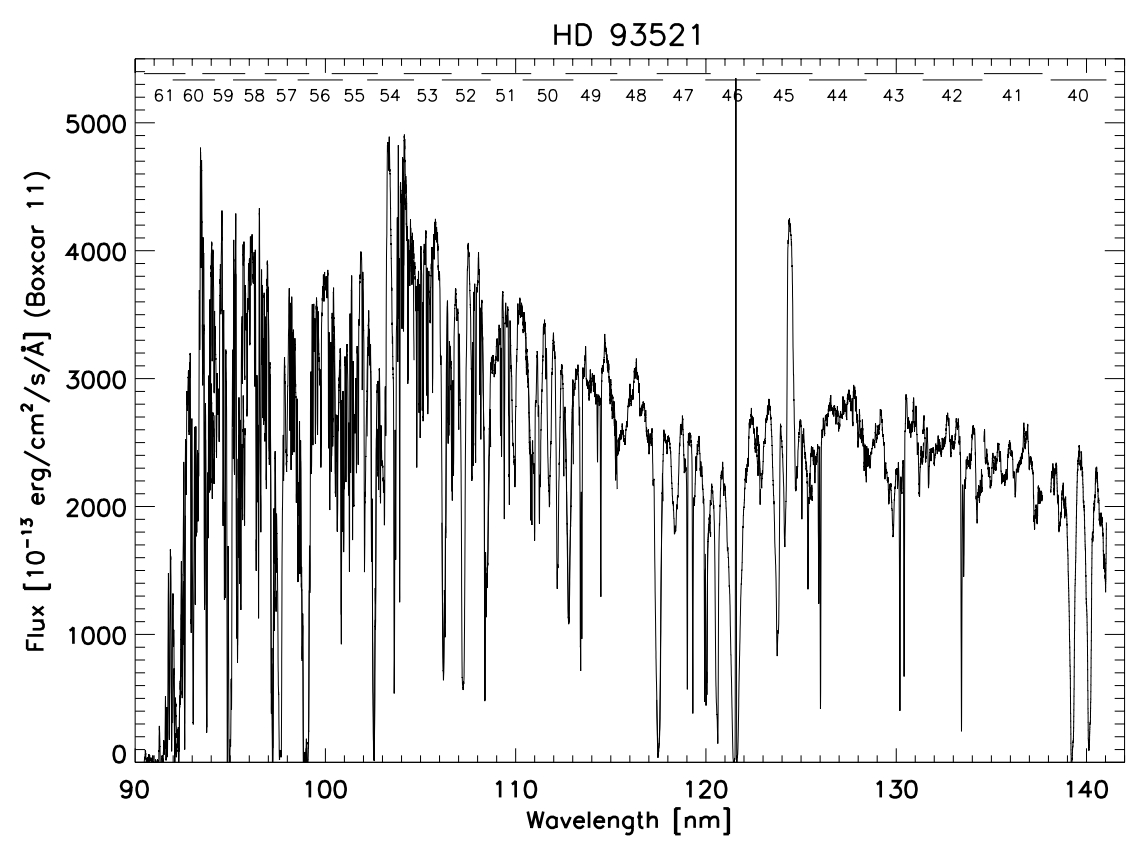

Fig. 8. ORFEUS II Echelle spectrum of HD 93521. The individual Echelle orders are plotted separately, the wavelength range for each order is indicated at the top of the figure. Except for orders 40/41 and 41/42 there is an overlap region between adjacent orders which increases with the order number. The spectrum is plotted with an 11 pixel wide boxcar smoothing

Grewing M., Appenzeller I., Barnstedt J., et al., 1998, in: Ultraviolet Astrophysics, Beyond the IUE Final Archive, ESA SP-413, 757

Hurwitz M., Bowyer S., 1986, Proc. SPIE 627, 375

Hurwitz M., Bowyer S., 1996, in: Astrophysics in the Extreme Ultraviolet, Bowyer S. and Malina R.F. (eds.). Kluwer Acad. Publishers, p. 601

Hurwitz M., Bowyer S., Bristol R., et al., 1998, ApJ 500, L1

Jenkins E.B., Reale M.A., Zucchino P.M., Sofia U.J., 1996, Ap. Space Sci. 239, 315
Krämer G., Eberhard N., Grewing M., et al., 1988, in: A Decade of UV Astronomy with IUE, ESA SP-281, 2, 333

Krämer G., Appenzeller I., Bowyer S., et al., 1990, in: Observatories in Earth Orbit and Beyond, Kondo Y. (ed.). Astrophysics and Space Science Library Kluwer Acad. Publishers, 166, p. 177

Martin C., Jelinsky P., Lampton M., Malina R.F., 1981, Rev. Sci. Instrum. 52, 1067

Schroeder D.J., Hiliard R.L., 1980, Appl. Opt. 19, 2833 